\title{
AN INSTRUMENT FOR MEASURING THE MOMENTUM FLUX FROM ATOMIC AND CHARGED PARTICLE JETS
}

\author{
S. A. Cohen, F. Zonca, J. Timberlake, T. Bennett, \\ J. Cuthbertson, W. Langer, and R. Motley \\ Plasma Physics Laboratory \\ Princeton University \\ Princeton, N. J. 08543
}

\section{DISCLAIMER}

This repon was prepared as an account of work sponsored by an agency of the United States Government. Neither the United States Government nor any agency therecf, nor any of their employes, makes any warranty, express or implied, ot assumes any legal liability or responsibility for the accuracy, completeness, or usefulness of asy information, apparatus, product, or process disclosed, or rcpresents that iss use would not infringe privately owned rights. Reference herein to any specific commercial product, process, or service by trade name, trademark, manufacturer, or otherwise does not necescarily constitute or imply tis endorsement, reconmendation, or favoriag by the United Stule Government or any agency thereof. The views and opinions of authors expressed herein do not necessarily state or reflect those of the United States Governmest or any agency thereaf. 


\begin{abstract}
We have developed an instrument to measure the momentum flux from an intense plasma stream for which the standard techniques used for low pressure gases $<<10$ Torr) at room temperature are unsuitable. This device, a Plasma Momentum Meter, can measure forces of $10^{-5}-10^{3}$ Newtons with a response time of $<50$ ms onto surfaces of different materials immersed in dense plasmas $\left(\mathrm{n}>10^{12} \mathrm{~cm}^{-3}\right.$ ). Such forces are transmitted predominantly by ionic and neutral species, with 10's of eV's of kinetic energy, are accompanied by high heat fluxes, and are pulsed. The momentum flux onto a biasable target plate is transferred via a suspended quartz tube onto a sensitive force transducer, a capacitance-type pressure gauge. This protects the transducer from thermal damage, arcing and spultering. An absolute force calibration of the PMM to $1 \%$ accuracy has been made and is described. A flat carbon target has been used in measurements of the momentum flux: of $\mathrm{He}, \mathrm{Ne}, \mathrm{Ar}$, and $\mathrm{Kr}$ plasmas produced in a magnetized linear plasma device.
\end{abstract}




\section{Introduction}

To perform quantitative studies on the interaction between atomic, ionic, and/or plasma jets with solid or liquid material surfaces of varying shapes, it is necessary to measure all the moments of the Boltzmann equation - particle, momentum, and energy fluxes being the 3 lowest order moments - for both the incoming and reflected beam. Measurements of density of room temperature guses or gas jets at low pressure $\quad<10$ Torr) are commonly performed by ionization, thermocouple, or manometer gauges. From the measured density, the kinetic theory of gases allows accurate calculations of momentum and energy flows and pressure. However, the above-named measuring techriques are generally unsuitable for surfaces immersed in dense plasmas $\left(n>10^{12}\right.$ $\mathrm{cm}^{-3}$ ); little experimental data is available on the effects of ionic impacts, in the erergy range $<100 \mathrm{eV}$, onto surfaces; and the theory of plasmas is sufficiently complex (due, for example, to varying collisionality) that single-point measurements of plasma temperature and density in the plasma interior, as by probes or Thomson scattering, do not readily yield an accurate prediction for the particle, momentum, or energy fluxes at the boundaries of the plasma. To address part of this problem, we have developed a diagnostic instrument to measure the absolute pressure (momentum flux) from an intense plasma stream where forces onto surfaces defining the plasma boundary are transmitted by ionic particles with 10's of eV's of kinetic energy, are accompanied by high heat fluxes, are pulsed, and are also transmitted from neutral species within the plasma. We refer to this device as a Plasma Momentum Meter (PMM). ${ }^{1}$

The PMM has several additional capabilities: it can serve as a bolometer or Langmuir probe; it can be used to infer sheath potential without the need to measure electron temperature; and it is suitable for measuring momentum and energy reflection coefficients of material surfaces. These phenomena are of great interest in fusion research, particularly in the design of divertor systems for magnetic confinement devices such as ITER. ${ }^{2}$

\section{Concept and Construction}

The pressure transducer element of the PMM is a Baratron ${ }^{\circledR}$, commonly used as a pressure sensor in the range $10^{-6}$ to $10^{4} \mathrm{mTorr}$ for room temperature gases. The baratron is a capacitance manometer, i.e., a pressure differential causes elastic deflection of a thin, tensioned, circular, metallic diaphragm which is one electrode of a capacitor. From the specifications ${ }^{3}$ of the head used, model 317HS MKS rated to 1 Torr full-scale, forces in the range $10^{-5}-10^{-1}$ Newtons can be measured with an accuracy of $1 \%$, once the conversion from pressure to force has been obtained. (According to the manufacturer, the gauge output, $X$, has an uncertainty $<1 \%$ at $-10 \mathrm{mTorr}$ and $<0.3 \%$ at $>100 \mathrm{mTorr}$.) This conversion requires knowledge of the effective area of the metallic diaphragm or an absolute calibration, to be described later. Use of baratron heads with higher pressure capabilities, to $10^{4}$ Torr, allows extension of the PMM force range to 1000 Nts. 
Other pressure transducers, such as piezoelectric or strain gauges or torsion pendula, may be used. However, they are less suitable for our application in an intense magnetic field and pulsed plasma environment.

Energetic atomic/ionic/plasma jets must not come into intimate contact with the baratron pressure sensor because of the thinness and fragility of its diaphragm. Rather, the momentum flux contacts a refractory target plate and is transmitted to the diaphragm via a quartz tube with an i.d. of $3 \mathrm{~mm}$, an o.d. of $5 \mathrm{~mm}$, and a length of 68.5 $\mathrm{cm}$. Quartz tubing was chosen because of its electrical insulating properties, light weight, rigidity even at elevated temperatures, and low thermal conductivity. ${ }^{4}$ Calculations showed that, even after one hour of plasma bombardment of the target at $10 \mathrm{~W} / \mathrm{cm}^{2}$, the temperature rise of the quartz tube at the diaphragm would be less than $30 \mathrm{~K}$ and that the increase in length of the tube would not alter the calibration. The hollow quartz tube provides a channel to run wires to the front refractory target. This allows for electrical biasing of the target or monitoring the target temperature with a thermocouple. In applications where a magnetic field, B, is present, it is advisable to orient these wires parallel to $B$ to avoid magnetic forces.

The carbon target is manufactured from ATJ graphite. It is shaped as $\mathbf{2}$ coaxial discs, roughly 1 and $3.5 \mathrm{~cm}$ in diameter, each $0.5 \mathrm{~cm}$ thick. The smaller disc has a coaxial hole $5 \mathrm{~mm}$ in diameter which allows it to be securely placed onto the quartz tube. The diameter of the larger disc was chosen to exceed the diameter of the plasma jet by about $1 \mathrm{~cm}$. Targets of different materials, shapes, and orientations may be substituted for the carbon one.

The quartz tube has a second carbon plug on the end which presses against the diaphragm. (It is similar in shape to the carbon target which faces the plasma.) This distributes the force more evenly over the diaphragm, preventing plastic distortion. Between the carbon plug and the diaphragm is a proprietary material cushion which reduces bouncing of the quartz rod off the diaphragm when an impulsive load is applied.

The tube is maintained in a horizontal position by means of two pairs of $\sim 3 \mathrm{mil}$ chromel wires that suspend the tube at points about 10 and $30 \mathrm{~cm}$ from the target and plug ends, respectively. (See Fig. 1.) The exact location of each support was chosen to avoid integral or fractional length resonances in tube bending. At each suspension point the wires are at $45^{\circ}$ with respect to the vertical. Each pair of wires is co-planar and perpenditular to the axis of the tube. The length of each wire, controlled by micrometer-type snounts, is about $10 \mathrm{~cm}$. This suspension allows only longitudinal, axial displacements of the quartz tube. A thin metal shield in front of the PMM protects the wires from plasma bombardment.

The quartz tube and wires are mounted in a stainless-steel vacuum housing with port crosses for the micrometer-type mounts. The baratron is attached to one end of the vacuum housing by use of stainless-steel welded bellows. The length of the bellows is also set with a micrometer mount. This is used to control the contact between the carbon plug, the damping cushion, and the diaphragm and is an essential element in the calibration routine.

The output of the pressure sensor, $\mathrm{X}$, the force, $\mathrm{F}_{\mathrm{x}^{\prime}}$ exerted on the target plate by 
the plasma column and the plasma momentum flux, $\Phi$, are related by

$$
\Phi=\mathrm{F}_{\mathrm{x}} / \mathrm{S}_{\text {plasma }}=X \mathrm{~S}_{\text {eff }} / \mathrm{S}_{\text {plasma }},
$$

where $S_{\text {eff }}$ is the effective interaction area of the diaphragm and $S_{\text {plasma }}$ is the plasma cross section. For a circular tensioned diaphragm, deformed by a uniformly applied force, $S_{\text {eff }}$ can be easily calculated by analytic methods. But, because the exact shape of deformation caused by the contact of the carbon plug is not readily measured, the PMM needs to be calibrated to experimentally determine $S_{\text {eff }}$.

\section{Calibration}

\section{A. Force}

Calibration may be accomplished by several different methods, many of which rely on gravity. The simplest method we have found is to move the baratron axially relative to the quartz rod using the bellows connection between the baratron and the vacuum tube. When the baratron is moved towards the quartz rod, the angle, $\theta$, of the support wires with respect to the vertical increases, see Fig. 1b. The resulting increment in gravitational force, $\Delta F_{g}$ is related to the displacement, $\Delta x$, of the quartz tube by

$$
\Delta \mathrm{F}_{\mathrm{g}}=\mathrm{M} \omega_{\mathrm{o}}{ }^{2} \Delta \mathrm{x}=\mathrm{Mg} \sin \theta \text {, }
$$

where $g$ is the gravitational acceleration, $M$ is the total mass of the quartz tube and attachments, e.g., the carbon plug, carbon target, and electrical wires, and $\omega_{0}$ is the free oscillation frequency of the pendulum. The mass was measured using a precision microbalance; the natural frequency was measured using a pickup coil near the carbon target and a small, < 0.05 gram, magnet, temporarily attached to the target. The tota! mass of the pendulum and the pendulum frequency were found to be $34.255 \pm 0.001$ grams and $16.16 \pm 0.02 \mathrm{~Hz}$, respectively. The force increment is related to the PMM output by

$$
\Delta F_{g}=\Delta \times S_{\text {eff }} .
$$

We define a PMM response,

$$
R=\Delta X / \Delta x
$$

from the gravitational loading, giving

$$
\mathrm{S}_{\mathrm{eff}}=\mathrm{M} \omega_{\mathrm{o}}^{2} / \mathrm{R} .
$$


From a least squares fit of the measured baratron signal versus baratron displacement data shown in Fig. 2, we get $R=103.06 \mathrm{mTorr} / \mathrm{mm}$ (with $\sigma_{R}=0.47$ $\mathrm{mTorr} / \mathrm{mm}$ ) or $\mathrm{R}=1.374 \times 10^{3}$ dyne $/ \mathrm{cm}^{3}$ (with $\sigma_{\mathrm{R}}=6 \mathrm{dyne} / \mathrm{cm}^{3}$ ), from which $\mathrm{S}_{\text {eff }}=$ $6.51 \pm 0.04 \mathrm{~cm}^{2}$. (The geometrical area of the diaphragm is about $20 \mathrm{~cm}^{2}$.) This calibration yields the conversion of gauge pressure to applied force (both in practical units),

$$
F_{x}(\text { grams })=8.86 \pm 0.05 \times 10^{-3} \times(\text { mTorr })
$$

To ohtain the momentum flux, the cross sectional area of the plasma must be known, Eq. (1). As described later, the plasma we have studied is not uniform, thus our measurements of momentum fluxes are averages over the plasma cross section.

The baratron pressure data in Fig. 2 start at $\sim 30$ mTorr. This value is a fixed offset applied to the baratron by shortening the length of the bellows section by use of the micrometer positioning mechanism. It is useful to preload the cushion between the carbon plug and the diaphragm to reduce compression during subsequent loads because motion of the quartz rod causes a reduction in force applied to the baratron by an amount $\mathrm{Mg} \sin \theta$. Smaller preloads are to be used for detecting smaller forces.

It should be noted that this method of calibration allows in situ recalibrations, without the need to vent the PMM to air. Also, calibrations done with the PMM exposed to air require a baratron head with a pumpable chamber on the back face of the deformable diaphragm. Without this pumpable chamber, exposure to atmospheric pressure causes the diaphragm to seat itself on the counter electrode.

\section{B. Response Time}

The variable capacitor comprising the baratron is part of an if capacitance bridge which operates at $10 \mathrm{kHz}$. The high accuracy of the baratron requires an electronic averaging time of typically 10 cycles. This gives an intrinsic response time of $1 \mathrm{~ms}$. (The response of the baration to gas pressure changes can be longer due to vacuum conductances.)

The response time of the PMM is longer, however, due to the natural frquency and mass of the quartz rod, the natural frequency of the diaphragm, and the small, but finite, compression of the damping cushion placed between the diaphragm and the carbon plug. The compression of the cushion is approximately linear with applied force at low forces, but rapidly saturates at higher forces. From the nonlinearity of the data (Fig. 2) at $x--55.35 \mathrm{~mm}$, we estimate that the compression saturates at a $F_{s}<0.001$ grams, corresponding to a displacement of $\left(F_{s} / M \omega_{0}\right) \sim 0.001 \mathrm{~cm}$. This will add less than $1 / 4$ an oscillation period to the response time of the PMM, corresponding to $\sim 75$ ms for the undamped pendulum and $<30 \mathrm{~ms}$ for the damped pendulum.

The response time of the PMM was measured by using plasma pulses of varying duration from a linear plasma device, (described in more detail in Sec. $N$, see Fig. 3 ). 
Short duration plasma pulses, $<20 \mathrm{~ms}$, provide a current to the carbon target as shown in Fig. 5a. The force is essentially impulsive, followed by a decay time of $\sim 10 \mathrm{~ms}$. The PMM output rises to a peak about $25 \mathrm{~ms}$ after the impulse and then decays in a similar time.

Plasma pulses of longer duration have a more complicated time evolution. Again there is a rapid rise, $\sim 1 \mathrm{~ms}$, in plasma current to the carbon target. This is followed by the same $\sim 10 \mathrm{~ms}$ decay to a valley roughly equal to $1 / 4$ the peak value. This is followed by a rise in plasma current in $30 \mathrm{~ms}$ to a plateau which is about twice the amplitude of the valley. Measuring from the time of breakdown of the gas, the formation of the plasma takes about $50 \mathrm{~ms}$ to stabilize at the plateau level. When the plasma is terminated, the current drops to zero in about $1 \mathrm{~ms}$. From that instant, the PMM signal falls to 1/e of its peak in $50 \mathrm{~ms}$, as shown in Fig. $5 \mathrm{~b}$ for plasma pulses of 2 different durations.

\section{Plasma Device}

The apparatus used for testing the PMM is a Lower Hybrid Coaxial Plasma Device ${ }^{5}$, which is a pulsed and magnetized plasma column. The coaxial plasma source is driven by $1 \mathrm{~kW}$ of $\mathrm{rf}$ power at $2.45 \mathrm{GHz}$ applied to a center conductor which acts as a quarter wave antenna. The plasma source produces an intense plasma column of about $1 \mathrm{~cm}$ radius and 10 to $>200 \mathrm{~ms}$ duration, depending on the power supply and $\mathrm{rf}$ feedthrough capabilities. A magnetic field of $\sim 3.5 \mathrm{kG}$, essential to the propagation and absorption of lower hybrid waves, is provided by two sets of coils arranged in a Helmholtz-type configuration. A schematic of this device is shown in Fig. 3.

The rf power propagates through the plasma as lower hybrid waves. The absorption length for the lower hybrid waves is typically $<10 \mathrm{~cm}$. Damping is predominantly on the electrons. 5

Plasma parameters obtained depend on gas pressure, applied if power, as well as bias supplied to a neutralizer plate, e.g., the target of the PMM, downstream from the source. If the neutralizer plate is biased positively with respect to plasma potential, electrons are more rapidly lost from the plasma column, reducing the plasma density. Plasma parameters are obtained with a scanning Langmuir probe. For the parameter range of this device, the shape of the probe characteristic is well described by magnetized probe theory. ${ }^{6}$

For the gases $\mathrm{He}, \mathrm{Ne}, \mathrm{Ar}$, and $\mathrm{Kr}$, the electron density typically varies from $\mathrm{n}_{\mathrm{e}} \sim \mathbf{1 0}^{13}$ $\mathrm{cm}^{-3}$ for $\mathrm{He}$ to $\mathrm{n}_{\mathrm{e}} \sim 7 \times 10^{13} \mathrm{~cm}^{-3}$ for $\mathrm{Kr}$. The temperature $\mathrm{T}_{\mathrm{e}}$, varies from $\sim 10 \mathrm{eV}$ for He to $-3 \mathrm{eV}$ for $\mathrm{Kr}$ discharges. The more massive gases produce denser, colder plasmas. The ion temperature, $T_{i}$, has not been measured. Calculations based or classical thermal equilibration indicate that $T_{i} \leq 1 \mathrm{eV}$. The $n_{e}$ profile is approximately triangular while the $T_{e}$ profile is flat; the $T_{i}$ profile is expected to be broad (see Fig. 4). 
The system is pumped by a $1500 \mathrm{l} / \mathrm{s}$ turbo pump, capable of maintaining pressures of $<10^{-6}$ Torr in the vacuum vessel. During plasma operation, the typical operating pressure is a few mTorr corresponding to a neutral density of $-10^{14} \mathrm{~cm}^{-3}$. The gas handling system consists of two completely independent gas lines which enter the coaxial source. One of the two lines is connected to the vacuum vessel through a pressure regulating needle valve; the other through a piezoelectric valve for pulsed operation.

Charge exchange can be important in these plasmas because the neutral gas density is high, particularly near the plate where neutralized atoms are re-emitted. The residence time of implanted ions is not known. In the case of $\mathrm{He}$, few atoms are reionized after reflection from the target (or neutralizer plate) because of the long ionization length and short charge exchange length. Therefore, for helium, an appreciable amount of momentum flux may be attributed to neutrals.

\section{Measurements of Plasma Momentum Flux}

The PMM was installed in the $2.45 \mathrm{GHz}$ Lower Hybrid plasma source chamber facing the plasma source. The target plate of the PMM serves the function of a neutralizer plate. The PMM was electrically configured with a $1.130 \pm 0.005 \mathrm{ohm}$ resistor connected in series with a Kepco model 850 Bipolar Operational Amplifier, the target plate, and ground. The RF plasma potential is also referenced to the chamber which is at ground potential.

The bias voltage between the target plate and the chamber was measured with a Beckman Circuitmate DM40 digital voltmeter. The voltage across the resistor gives the total plasma current; it is displayed on an oscilloscope along with the signal from the baratron. Figure $5 b$ shows data from two sequential plasma pulses of different durations. The oscilloscope is triggered at the ime of rf turn on.

For a Ne gas plasma and a target bias of +15.0 volts, current signals, $A_{1}$ and $A_{2}$ of Fig. $5 b$, were displayed on a 1 volt/div scale and the respective baratron signals, $B 1$ and $\mathrm{B} 2$, were displayed on a $50 \mathrm{mV} /$ div scale. When the plasma was started, the current first rises sharply, then falls for $\sim 20-30 \mathrm{~ms}$, then rises to a a steady value at about $50 \mathrm{~ms}$. At plasma turn off, this signal returned abruptly to its beginning value. The reproducibility of the baratron signal was excellent. When one run was overlaid over another run with equivalent parameters, the baratron signais were virtually identical.

For this condition, the steady-state plasma current was $\sim 4 \mathrm{~A}$. Using the calibration described in Sec. III, the steady-state value of baratron output was converted to momestum flux, which is plotted vs. the bias voltage for $\mathrm{He}, \mathrm{Ne}, \mathrm{Ar}$, and $\mathrm{Kr}$ in Fig. 6 . Comparison of these data with probe theory and kinetic models will be described in a subsequent publication. ${ }^{7}$ Briefly, we note that the measured momentum flux for the most collisional plasmas agreed with that predicted by sheath theory.

After testing the PMM, it was removed from the plasma chamber and examined. Damage to the carbon target, attiributed to sputter erosion of the graphite by energetic ions, was clearly discernible (Fig. 7). 
In summary, a device for measuring pulsed or steady-state momentum fluxes in the harsh environment of intense magnetized plasnas has been developed. This device has been used to measure monentum fluxes to carbon surfaces inserted in the plasma flow and oriented normal to the flow direction.

\section{Acknowledgements}

This work supported by DOE contract No. DE-AC02-CHO-3073.

\section{References}

1. Patent pending

2. K. Tomabechi and the ITER Team, Plasma Physics and Controlled Nuclear Fusion Research 1988 (Proc. 12-th Conference, Nice, 1989) Vol. 3, IAEA-CN-50/F-I-4, 215.

3. MKS Instruments, Inc, "Baratron ${ }^{\circledR}$ specifications" , Burlington, MA.

4. Heraeus Amersil Inc.," Properties of Fused Quartz and Silica", 100 Heraeus Blvd. Buford GA.

5. R. W. Motley, S. Bernabei, and W. M. Hooke, Rev. Sci. Instrum. 50 (12), (1979) 1586.

6. J.G. Laframboise, UTIAS Report No. 100, Institute for Aerospace Studies, University of Toronto (1966).

7. F. Zonca, S.A. Cohen, J. Timberlake, et al., to be published in J. Nucl. Mater. Dec 1990. 


\section{FIGURE CAPTIONS}

Fig. 1. a) Photograph of the Plasma Momentum Meter showing the construction. b) Schematic of the Plasma Momentum Meter showing the parts as in the photograph above. The parts are: A) front carbon target and rear carbon plug, B) adjustable supports for horizontal positioning and calibration, $C$ ) suspension wires for free horizontal axial motion, D) quartz tube for thermal insulatien and rigid force transmission, E) thermocouple leads for temperature monitoring an.d biasing target plate, F) MKS Baratron ${ }^{(B)}$ sensor, G) capacitance bridge for pressure measurements, H) vacuum housing, and J) protective shield for suspension wires.

Fig. 2. Calibration data obtained to calculate the effective surface area, $\mathrm{S}_{\mathrm{eff}}$ of the diaphragm.

Fig. 3. Schematic of the $2.45 \mathrm{Ghz}$ lower hybrid plassma source used to test the Plasma Momentum: Meter.

Fig. 4. Typical profiles of electron density, $\mathrm{n}_{\mathrm{e}^{\prime}}$ electron temperature, $\mathrm{T}_{\mathbf{e}^{\prime}}$ and ion temperature, $T_{i}$ in the $2.45 \mathrm{GHz}$ lower hybrid plasma source. The plasma cross section is $1 \mathrm{~cm}$ in radius.

Fig. 5. a) Sketch of the response of the PMM for the impulse of a He gas plasma onto a negatively biased target. b) Oscilloscope displays for Ne gas plasmas of two different durations, $-120 \mathrm{~ms}$ and $\sim 260$ ms. The target bias was +15.0 volts with respect to the vacuum vessel. $A_{1}$ and $A_{2}$ are the net currents from the plasma to the target. $B_{1}$ and $B_{2}$ are the corresponding Baratron signal outputs for $A_{1}$ and $A_{2}$, respectively. The measured value is from the flattop of $B_{2}$. The three dashed traces are sketched to correspond to the actual signal. (The gradual downward slope of all the signals is caused by the lower hybrid plasma source magnetic field affecting the oscilloscope.)

Fig. 6. Results of the momentum flux vs. bias voltage for $\mathrm{He}, \mathrm{Ne}, \mathrm{Ar}$, and $\mathrm{Kr}$ plasmas.

Fig. 7. Photograph of the front carbon iarget plate of the Plasma Momentum Meter after testing in the $2.45 \mathrm{GHz}$ lower hybri "plasma source. The area of plasma impact is discernable on the plate. 


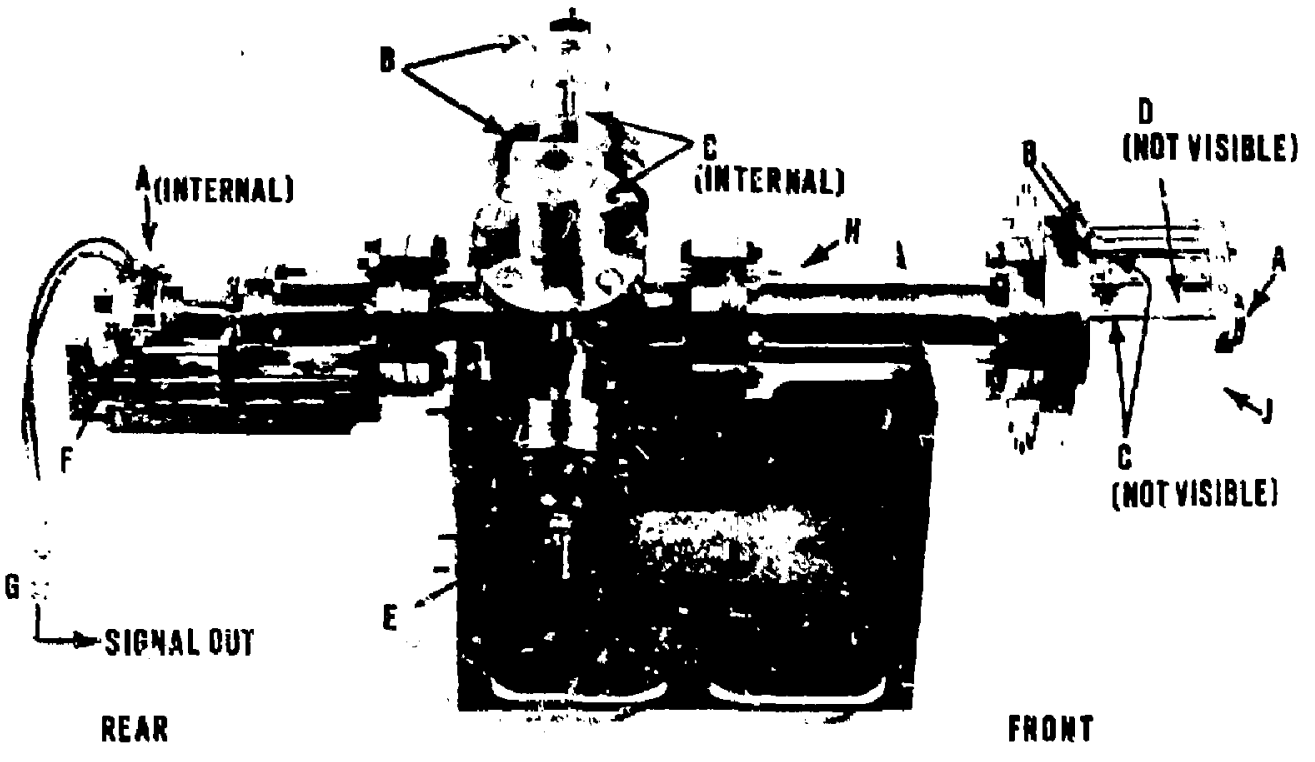

(b)

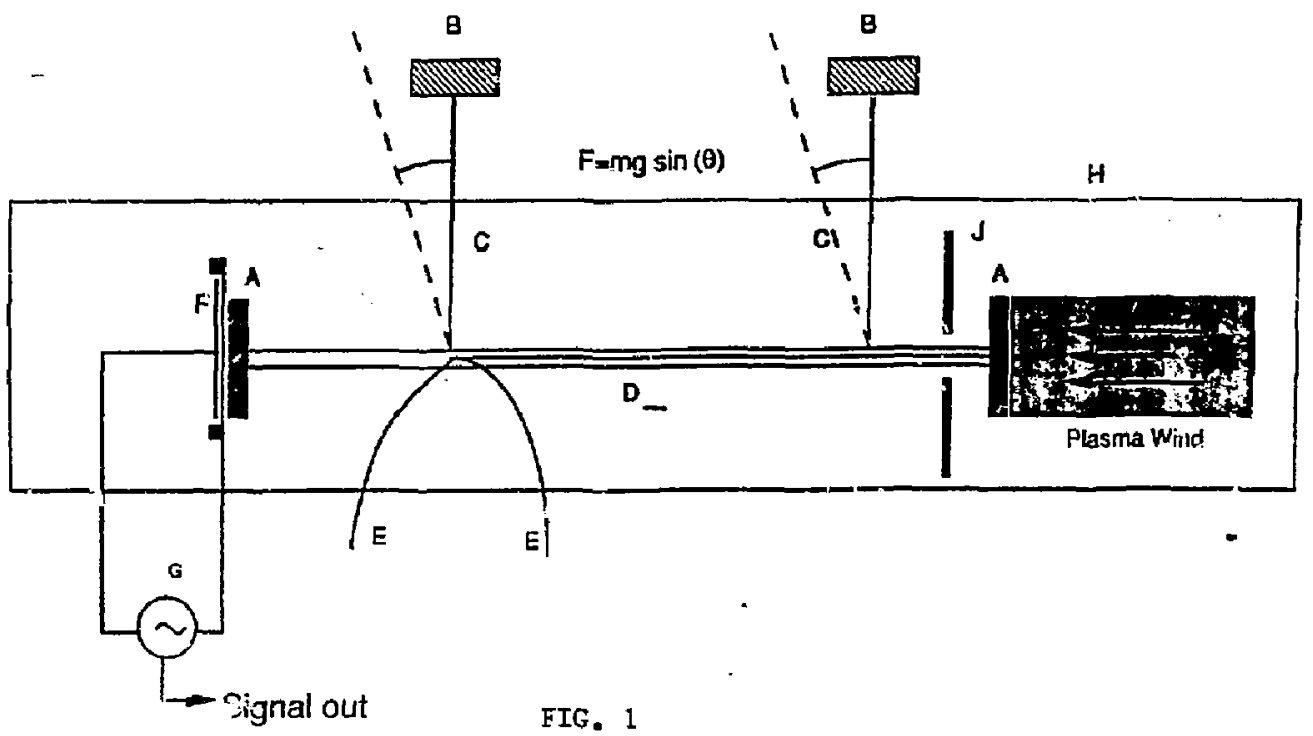




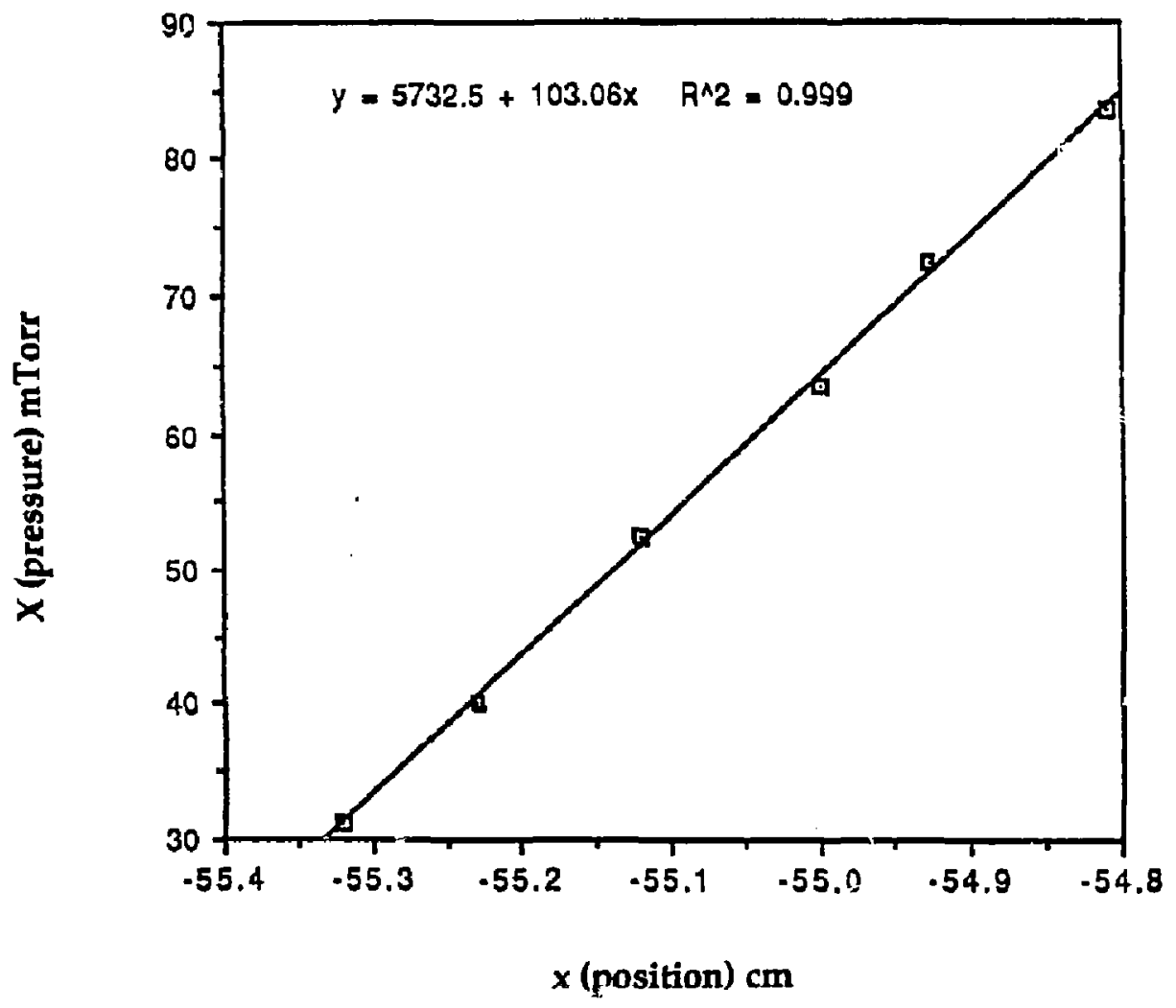

EIG. 2 


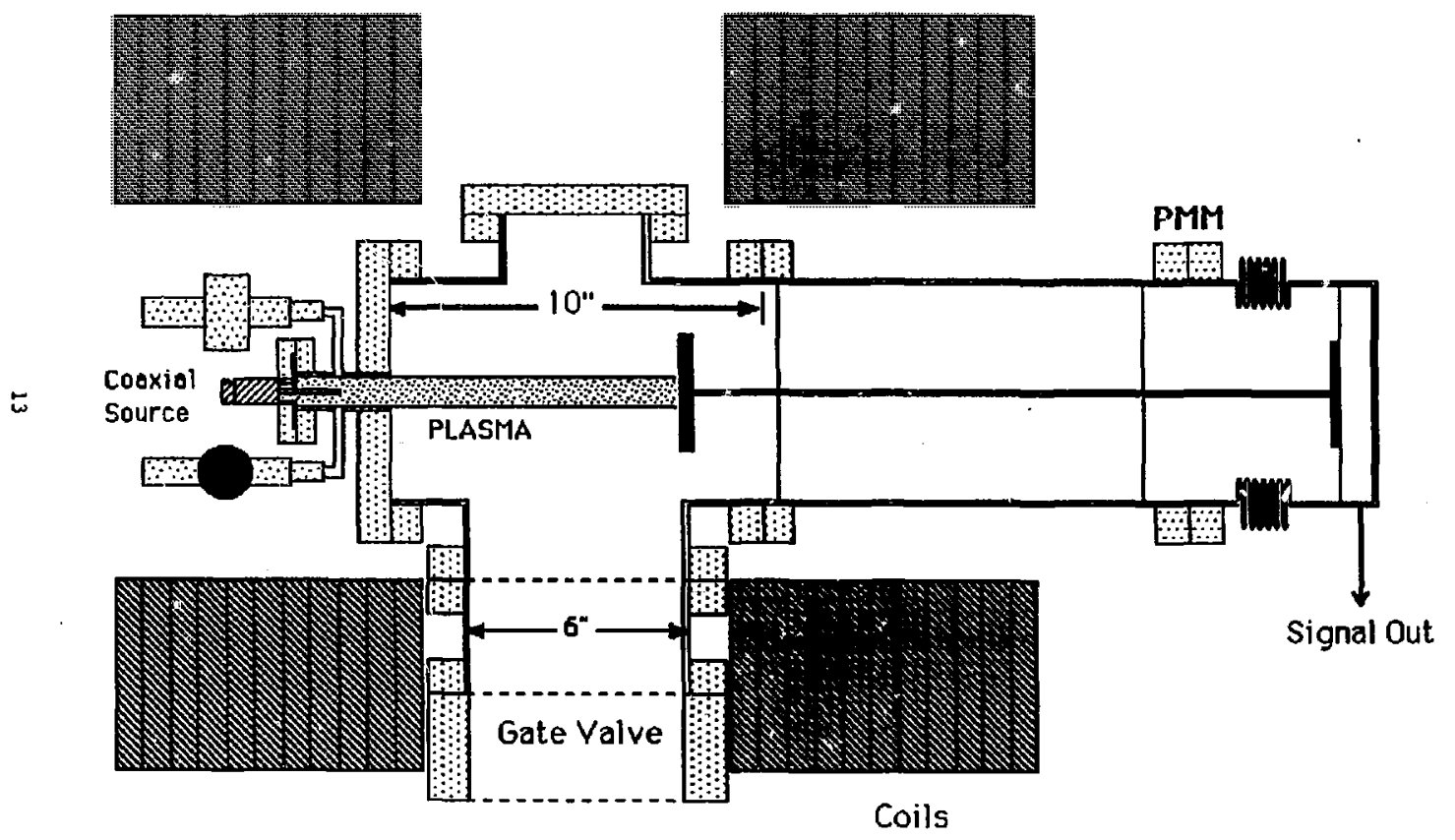

FIG. 3 

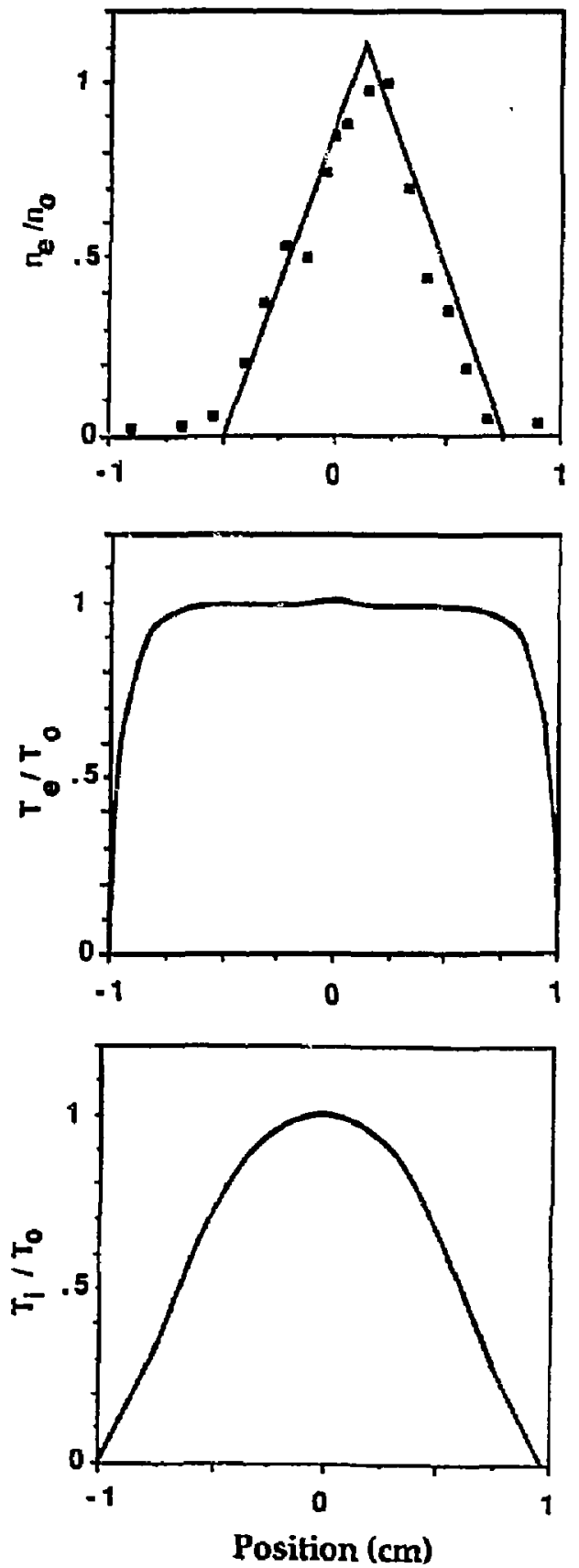

FIG. 4 

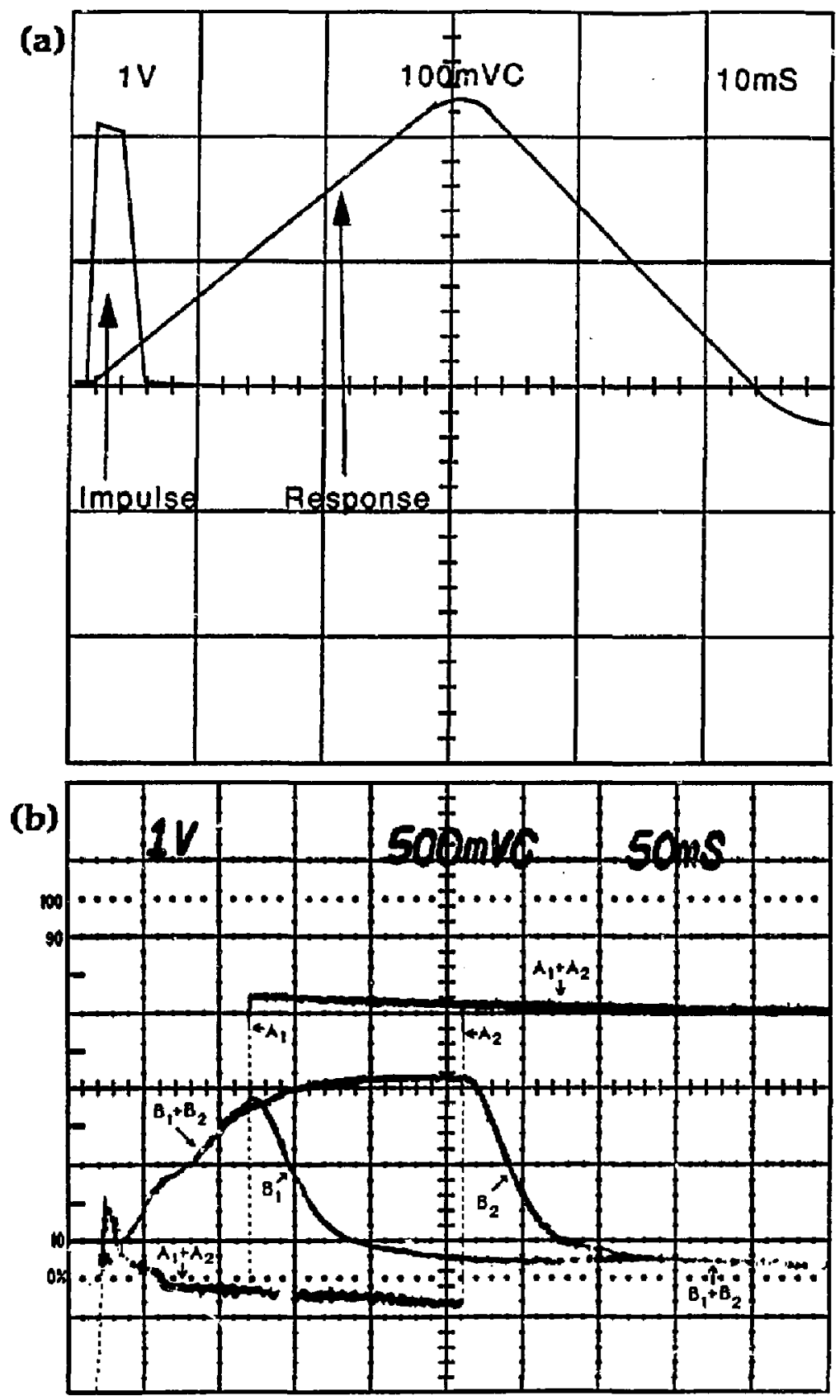

FIG. 5 


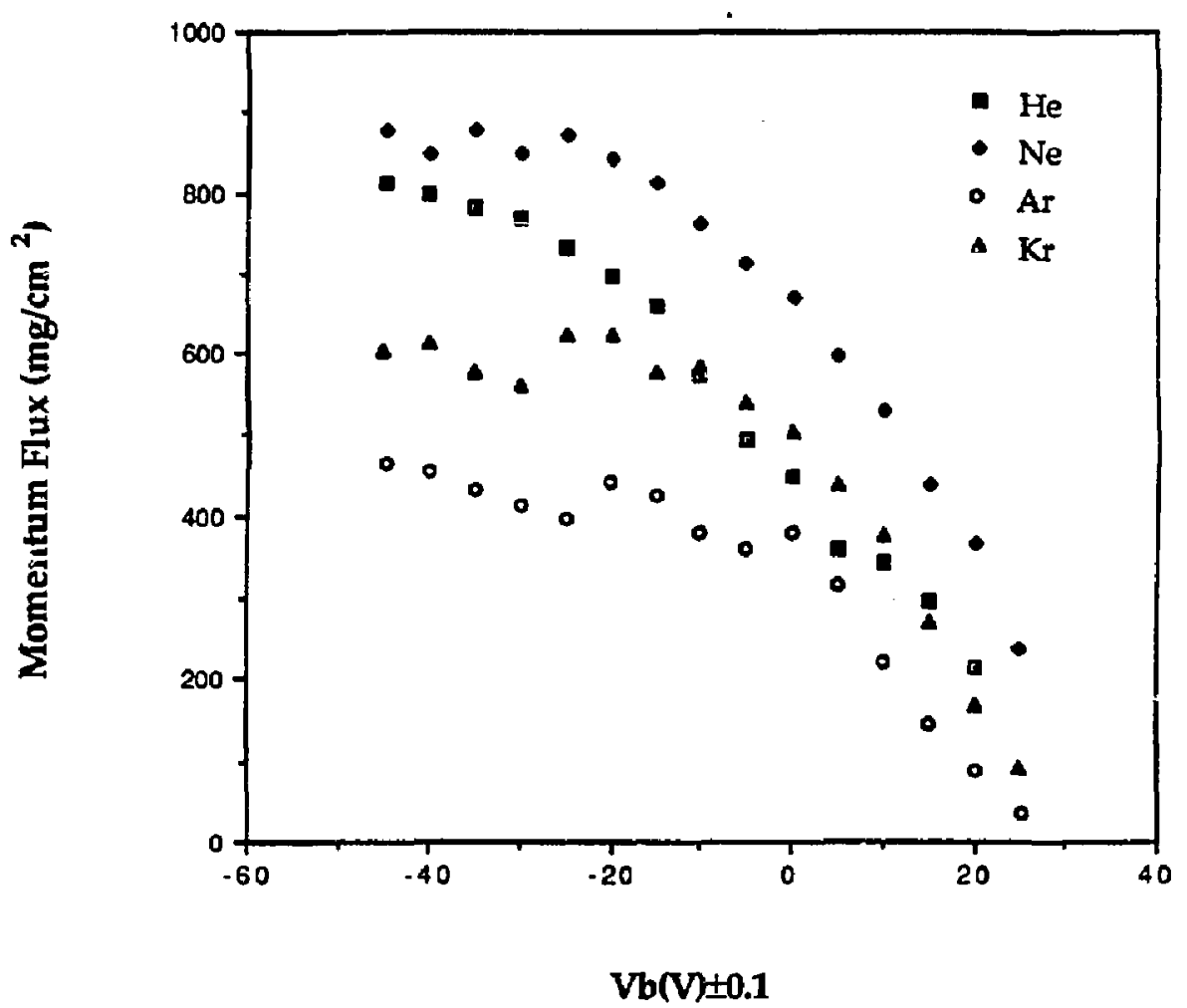

FIG. 6 


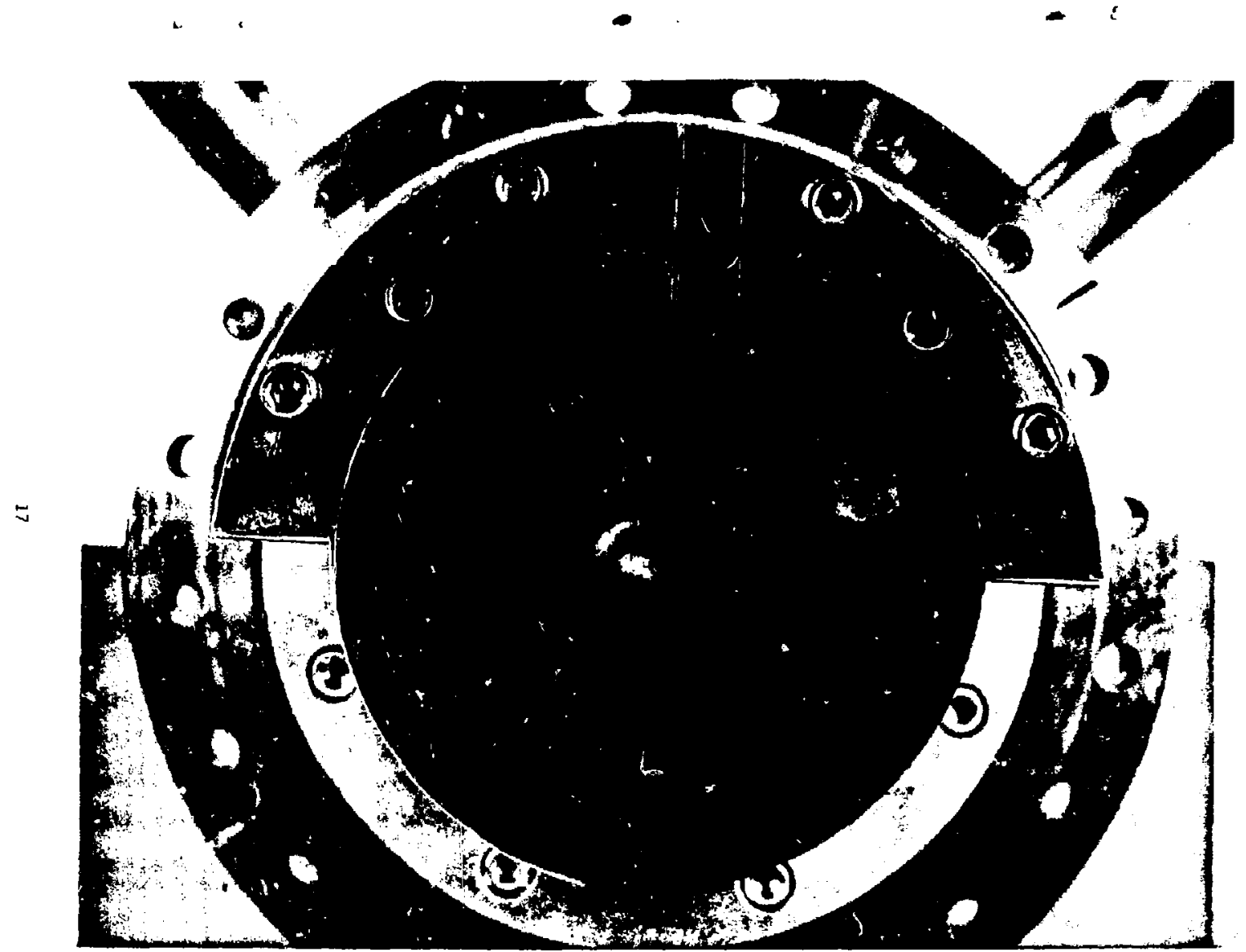

